\title{
PRIMER REGISTRO DE ÁCAROS ACUÁTICOS (ARRENURUS SP.) COMO ECTOPARÁSITOS DE ODONATOS EN LA HISPANIOLA
}

\author{
Daniel E. Perez-Gelabert ${ }^{1}$ y Francisco Alba Suriel ${ }^{2}$ \\ ${ }^{1}$ Integrated Taxonomic Information System (ITIS) and Department of Entomology, \\ National Museum of Natural History, Smithsonian Institution, P.O. Box 37012, \\ Washington, DC 20013-7012, USA.perezd@si.edu \\ ${ }^{2}$ Asociación de Fotógrafos de Naturaleza (ADFONA), Herrera, Santo Domingo Oeste, \\ República Dominicana. franciscoalba_64@hotmail.es
}

\section{RESUMEN}

Se registra por primera vez para la República Dominicana y la Hispaniola ácaros acuáticos del género Arrenurus Dugès, 1834 parasitando odonatos. El reporte se basa en un solo individuo de la damisela Telebasis dominicana (Selys, 1857) parasitado por múltiples individuos del ácaro acuático Arrenurus sp.

Palabras clave: ácaros acuáticos, Arrenurus, Odonata, Hispaniola.

Title: First record of water mites (Arrenurus sp.) as ectoparasites of Odonata in Hispaniola.

\section{ABSTRACT}

Water mites of the genus Arrenurus Dugès, 1834 are recorded for the first time parasitizing odonates in the Dominican Republic and Hispaniola. The report is based on a single individual of the damselfly Telebasis dominicana (Selys, 1857) parasitized by multiple individuals of the water mite Arrenurus sp.

Keywords: water mites, Arrenurus, Odonata, Hispaniola.

Los ácaros que viven en el medio acuático son ectoparásitos comunes de insectos acuáticos o semiacuáticos tales como Collembola, Coleoptera, Odonata, Diptera, Trichoptera, Heteroptera y Plecoptera (Martin, 2008). En los odonatos las larvas de ácaros son foréticas en las ninfas, se mueven de la exuvia al adulto cuando ocurre la muda y como adultas son parasíticas de los odonatos adultos (Andrew et al., 2015). Este parasitismo puede afectar la longevidad y reproducción de los odonatos (Forbes, 1991).

Mundialmente se conocen más de 5000 especies de ácaros acuáticos clasificados en unas 40 familias. Dado que su conocimiento es todavía incipiente, se piensa que su diversidad total probablemente es mucho mayor. Ocupan una gran variedad de hábitats acuáticos y semiacuáticos, entre ellos hoquedades de árboles, fitotelmata, manantiales sulfurosos, cataratas, lagos profundos y aún el medio marino (Cook, 1974).

Hasta ahora la diversidad acarológica de la Hispaniola solo ha tenido inventarios limitados a grupos específicos, realizados de manera ocasional casi completamente por especialistas extranjeros luego de visitas esporádicas a la isla. Para todo el orden Acarina la lista anotada de los artrópodos de la Hispaniola (Perez-Gelabert, 2008) recogió un total de 112 especies en 67 géneros, la gran mayoría de ellas especies parásitas de plantas y vertebrados.

En la Hispaniola solo existen unos pocos trabajos sobre ácaros acuáticos (Lundblad, 1936; Cook, 1981; Botosaneanu \& Bolland, 1997). Lundblad (1936) en sus investigaciones de 
lagos en Haití, reportó entre otros ácaros acuáticos, tres especies del género Arrenurus Dugès, 1834 (A. birgei Marshall, 1903, A. megalurus Marshall, 1903 y A. valencius Marshall, 1903). De todos los ácaros acuáticos, Arrenurus es el género más diverso, con unas 800 especies con distribución cosmopolita excepto en la Antártica (Smit, 2010). Reportes recientes de este género en el área neotropical incluyen la descripción de cuatro nuevas especies de Cuba (Orghidan \& Gruia, 1977), la descripción de 10 nuevas especies encontradas en lagos de México (Cramer \& Cook, 1992), el hallazgo de una nueva especie que habita bromelias (fitotelmas) en Venezuela (Rosso de Ferradás y Fernández, 2001) y Arrenurus parasitando varias especies de odonatos en Brasil (Rodrigues et al., 2013).

El conocimiento de la fauna odonatológica en la Hispaniola está mayormente limitado a su alfa-taxonomía. La fauna viviente consiste en 67 especies (48 Anisoptera y 19 Zygoptera), con solo 7 especies endémicas de la isla (Perez-Gelabert, 2008). Entre las últimas contribuciones al conocimiento de Odonata en la Hispaniola, el trabajo de Flint et al. (2006) aportó datos sobre la distribución por provincias de muchas de estas especies. Más recientemente, Torres-Cambas et al. (2015a) describen la nueva especie, Hypolestes hatuey y también examinan la distribución de varias especies consideradas amenazadas en las Antillas Mayores (Torres-Cambas et al., 2015b). Poco se ha escrito sobre su biología, interacciones tróficas y parasitismo.

El presente reporte está basado en una agregación de 29 individuos de Arrenurus sp. encontrados sobreeldorsoy partelateral torácicadeunahembradeladamisela Telebasis dominicana (Selys, 1857) (Odonata: Zygoptera: Coenagrionidae; Fig. 1A-B). Este individuo fue fotografiado y luego colectado con una red entomológica mientras volaba entre la vegetación adyacente a un canal de riego cercano al pueblo de Los Negros, Prov. Azua. Esta especie es endémica de las Antillas Mayores y está ampliamente distribuida en la Hispaniola. Ha sido registrada para las provincias dominicanas de Barahona, El Seibo, La Altagracia, La Vega, Puerto Plata, San Cristóbal, San Juan, Azua, Dajabón, Independencia, Peravia, Sánchez Ramírez y el Distrito Nacional (Flint et al., 2006).

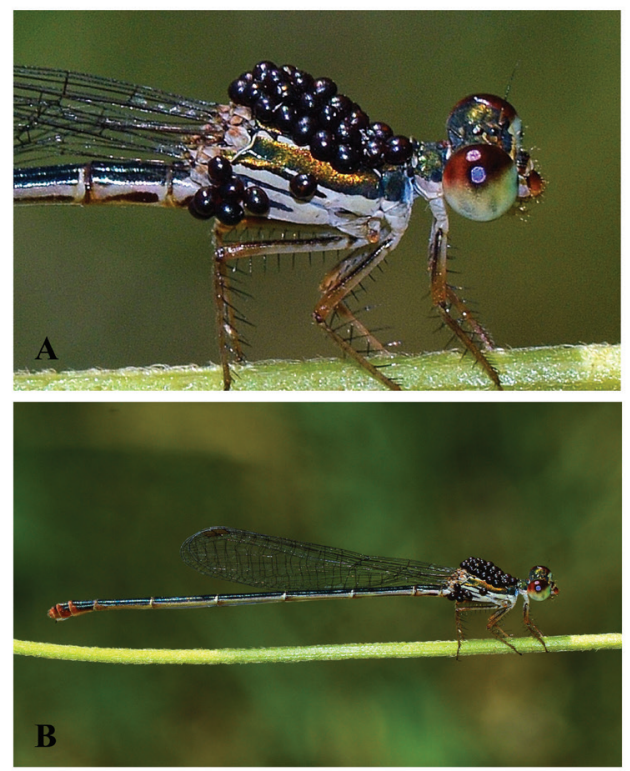

Figura 1A-B. Hembra de la damisela Telebasis dominicana (Selys, 1857), Odonata: Zygoptera, parasitada por 29 individuos de Arrenurus sp. (Acari: Hydrachnidia: Arrenuridae). 
En preparación para la producción de huevos y reproducción las hembras generan gran cantidad de vitelo rico en nutrientes en su hemolinfa. Ya que los ácaros se alimentan de hemolinfa al chupar a su hospedero, se piensa que los ácaros podrían preferir hembras en lugar de machos. En un caso que apoya esta idea, Andrew et al. (2015) encontraron que aunque ambos sexos eran parasitados en igual frecuencia, el número de ácaros parasitando las hembras era significativamente mayor. De manera similar, en el caso presente, la hembra de $T$. dominicana hospedera de Arrenurus sp. aparece parasitada por un alto número de ácaros.

El color de los ácaros está relacionado con la cantidad de hemolinfa ingerida y estos cambian de color progresivamente de anaranjado, verduzco a negro todos al unísono (Andrew et al., 2015). En el presente caso, los 29 ácaros encontrados sobre la hembra de $T$. dominicana mostraron un color negro uniforme y se observaban bien llenos de hemolinfa. Al igual que en otros casos, el tórax fue el lugar preferido por los ácaros para aferrarse al odonato hospedero.

Los ácaros acuáticos del género Arrenurus probablemente afectan a múltiples otras especies de odonatos encontrados en la Hispaniola. Sería instructivo investigar la distribución y frecuencia de este parasitismo en diferentes especies y en diferentes áreas geográficas de la isla.

Material estudiado. Una hembra de Telebasis dominicana (Selys, 1857) parasitada por 29 individuos de Arrenurus sp. (Hydrachnidia: Arrenuridae). REPÚBLICA DOMINICANA, Prov. Azua, cerca de Los Negros, 16/viii/2016, 18²2.707'N 7049.976'O, 18 msnm, cols. D. Perez-Gelabert y Francisco Alba S. Este material está depositado en la colección entomológica del National Museum of Natural History, Smithsonian Institution, Washington, DC, USA.

\section{AGRADECIMIENTOS}

La especie de damisela fue identificada por Oliver Flint, Jr. (National Museum of Natural History, Smithsonian Institution, Washington, DC). Santo Q. Navarro y Ruth H. Bastardo (IIBZ, República Dominicana) aportaron mejoras de escritura al manuscrito. Ruth H. Bastardo y Carlos de Soto Molinari (Santo Domingo) hicieron posible la expedición de campo.

\section{LITERATURA CITADA}

Andrew, R. J., P. R. Verma y N. R. Thaokar. 2015. A parasitic association of Odonata (Insecta) with Arrenurus Dugès, 1834 (Arachnida: Hydrachnida: Arrenuridae) water mites. Journal of Threatened Taxa, 7: 6821-6825.

Botosaneanu, L. y H. R. Bolland. 1997. A mite (Acari: Erythraeidae) as unusual parasite on an adult caddisfly (Trichoptera: Hydroptilidae) from the Dominican Republic (West Indies). Studies on the Natural History of the Caribbean Region, 73: 71-76.

Cook, D. R. 1974. Water mite genera and subgenera. Memoirs of the American Entomological Institute, 21: 1-860.

Cook, D. R. 1981. Amsterdam expeditions to the West Indian islands, report 11. New hyporheic water mites from Haiti. Bijdragen tot de Dierkunde, 51: 135-144.

Cramer, C. y D. R. Cook. 1992. New species of Arrenurus (Acari: Arrenuridae) from Mexican lakes. Acarologia, 33: 349-366. 
Flint, O. S., R. H. Bastardo y D. E. Perez-Gelabert. 2006. Distribution of the Odonata of the Dominican Republic. Bulletin of American Odonatology, 9: 67-84.

Forbes, M. R. L. 1991. Ectoparasites and mating success of male Enallagma ebrium damselflies (Odonata: Coenagrionidae). Oikos, 60: 336-342.

Lundblad, O. 1936. An investigation of some Hispaniolan lakes. (Dr. R. M. Bond's expedition) Über einige Hydracarinen aus Haiti. Arkiv for Zoologi, 28A: 1-30.

Martin, P. 2008. Water mites (Hydrachnidia, Acari) and insects: a survey of a seldom considered relationship. Entomologie Heute, 20: 45-75.

Orghidan, T. y M. Gruia. 1977. Quatre espèces d'Arrenurus de Cuba. En: Résultats des Expeditions biospéologiques cubano-roumaines à Cuba, 2: 231-240.

Perez-Gelabert, D. E. 2008. Arthropods of Hispaniola (Dominican Republic and Haiti): a checklist and bibliography. Zootaxa, 1831: 1-530.

Rodrigues, M. E., C. Carriço, Z. Texeira Pinto, P. Martins Mendonça y M. M. de Carvalho Queiroz. 2013. First record of Acari Arrenurus Dugès, 1834 as a parasite of Odonata species in Brazil. Biota Neotropica, 13: 365-367.

Rosso de Ferradás, B. y H. R. Fernández. 2001. Elenco y biogeografía de los ácaros acuáticos (Acari, Parasitengona, Hydrachnidia) de Sudamérica. Graellsia, 61: 181-224.

Smit, H. 2010. Two new species of the genus Arrenurus from Pohnpei, Federal States of Micronesia (Acari: Hydrachnidia: Arrenuridae). Zootaxa, 2006: 50-54.

Torres-Cambas, Y., M. O. Lorenzo-Carballa, S. Ferreira y A. Cordero-Rivera. 2015a. Hypolestes hatuey sp. nov.: a new species of the enigmatic genus Hypolestes (Odonata, Hypolestidae) from Hispaniola. Zootaxa, 4000 (2): 207-226.

Torres-Cambas, Y., A. D. Trapero-Quintana, M. O. Lorenzo-Carballa, D. Newell, C. Suriel, y A. Cordero-Rivera. 2015b. An update on the distribution of threatened odonate species from the Greater Antilles. International Journal of Odonatology, http://dx.doi.org/10.1080/ 13887890.2014 .928241$.

[Recibido: 30 de agosto, 2016. Aceptado para publicación: 26 de septiembre, 2016] 